\title{
Effect of Nano-Interface of Ru Underlayer in the Carrousel Sputtering Method
}

\author{
R. Imaizumi, M. Munakata, M. Ohkoshi*, K. Maki**, S.I. Aoqui \\ Energy Electronics Laboratory, Sojo Univ., 4-22-1 Ikeda, Kumamoto, Kumamoto 860-0082, Japan \\ Fax:81-96-326-3658, e-mail:g0911m03@cc.sojo-u.ac.jp \\ *Faculty of Computer Science, Kyushu Institute of Technology, 680-4 Kawazu, Iizuka, Fukuoka 820-8502, Japan \\ **Sumitomo Metal Mining Co., Ltd., 3-18-5 Nakakokubun, Ichikawa, Chiba 272-8588, Japan
}

\begin{abstract}
Increasing the uniaxial-magnetic anisotropy in thin-film materials is a key issue for micro-magnetic devices driven at a $\mathrm{GHz}$ frequency. An especially high uniaxial-magnetic anisotropy is known to have been induced by only the carousel sputtering technique. We analyzed the atomic deposition process of $\mathrm{Ru}$ nano underlayer by using Monte Carlo simulation with respect to sputtered particles. The results indicated that the nano-interface of the Ru underlayer had brought the further reduction in surface energy. It was found that the nano-interface of the underlayer remarkably contributed to the higher uniaxial-magnetic anisotropy of the films.

Key words : carrousel sputtering method, Ru underlayer, surface energy, nano-interface, higher uniaxial-magnetic anisotropy
\end{abstract}

\section{INTRODUCTION}

The sputtering method is one of effective plasma process technique to give nanostructural magnetic thin films according with their effective and characteristic properties.

In the so-called carousel sputtering method, a special deposition process of the sputtered atoms on the substrate has been already found for the developing the magnetic material operated at the high frequency of $\mathrm{GHz}[1,2]$.

In addition, a high uniaxial-magnetic anisotropy field $H_{k}$, which was five to ten times as high as those of the conventional films, it was found in the case of depositing of $\mathrm{Ru}$ underlayer by using only the same sputtering method [2]. It also had been known that this high uniaxial-magnetic anisotropy led to the highest magnetic resonance frequency more than $10 \mathrm{GHz}$ with high magnetic permeability as suitable materials for MMIC (Monolithic Micro-wave Integrated Circuit) device [3, 4]. However, the effect of the underlayer of the nano-interface on this film was not clear yet with respect to the uniaxial-crystalline orientation of the film.

In this study, it is reported that nano-interface of the underlayer can be effective to induce specially a sputtered atomic deposition by using Monte Carlo simulation with respect to surface energy of the underlayer.

\section{EXPERIMENTS}

2.1 Experimental Procedure

$\mathrm{Co}_{35} \mathrm{Fe}_{65}$ single layer and $\mathrm{Co}_{35} \mathrm{Fe}_{65} / \mathrm{Ru}$ double layers were prepared on glass substrate (Matsunami \#7050, $1 \times 45 \times 45 \mathrm{~mm})$ using carrousel sputtering method (RF-magnetron sputtering method) [4]. Ar gas pressures in deposition process were $3 \times 10^{-3}$ Torr. The thickness of the $\mathrm{Co}_{35} \mathrm{Fe}_{65}$ layer was $5000 \AA$ fixed in all films. The thickness of the underlayer of $\mathrm{Ru}$ was varied from 0 to 20 $\AA$. The sputtering targets for depositing the Co-Fe film were $\mathrm{Fe}$ and $\mathrm{Co}$ with 4 in.diam. and $5 \mathrm{~mm}$ thick. In this sputtering apparatus, the glass substrate was mounted on the side of the cylindrical electrode and was water-cooled. The cylindrical electrode was rotated about its central axis at 140 r.p.m., while target electrodes were placed opposite to the side of the cylindrical electrode. Atomic composition in the film was adjusted by changing input RF-power for each target. During the deposition time of one atomic layer for each target, the cylindrical electrode was set to be rotated by more than 4 times. Then, sputtered atoms from each target were deposited and mixed on the substrate without multilayer of $\mathrm{Co}$ and $\mathrm{Fe}$ formed [5]. For depositing the underlayer, Ru target with the same size as the $\mathrm{Co}$ and the $\mathrm{Fe}$ target were used.

The values of $H_{c}, H_{k}$, and $4 \pi M_{s}$ in as-deposited state were measured using a vibrating sample magnetometer (VSM). The structural characterization was carried out by an X-ray diffraction (XRD), using $\mathrm{Cu}-\mathrm{K} \alpha$ radiation, the $2 \theta$ scanning technique and Schultz method. 3 dimensional scanning electron microscope (3DSEM) was used to examine the microstructure of the coated underlayer surface.

\subsection{Procedure in the simulation}

The deposition of sputtered particles was analyzed by using Monte Carlo simulation method [6, 7]. This method has been known as an effective one which reflected actual behavior of sputtered atoms with collision and scattering as blow articles of four steps [7].

2.2.1 Divide into finite elements for the sputtering apparatus

Finite elements were setup the sputtering apparatus was composed by using 3DCAD (Computer Aided Design) and JMAG [8].

\subsubsection{Calculation of ejected particles from the target}

Distribution of ejected particles on the substrate was an important factor, because it drastically effect on the deposition process. This distribution was calculated by using the so-called cosine emission law: $1 / \cos \varphi$, where $\varphi$ 
is the angle between the ejected direction and the normal direction to the target. The ejected positions were obtained on the basis of the erosion formed by gas (argon) ion bombardment.

\subsubsection{Calculation of mean free path of sputtered particles} Mean free path of sputtered particles was very important to get the incident position, angle, and energy. In this simulation, the transport of the sputtered particles was calculated in an average of molecular and viscous flow regions. In the sputtering simulation, it has already known as a general method $[1,6,7]$. Therefore, the mean free path was calculated by $1 /(n \sigma)[\mathrm{m}]$, where $n$ is particle density and $\sigma$ is collision cross section, and Boltzmann distribution of velocity.

2.2.4 Calculation of collision and scattering angles between sputtered and gas particles

Perfectly elastic collision was assumed in it between sputtered particles and gas particles. The collision angle, which means incident direction of the gas particle to the sputtered particle, was assumed to exhibit its isotropic distribution. In parallel with this angle, the distribution of scattering angle was calculated by using the center-of-mass system.

\subsection{Setting of parameter values}

Ejected energy of the sputtered particle and the gas particles were set on $10 \mathrm{eV}$ and $1 \mathrm{eV}$, respectively, in which these parameters were estimated from experimentally measured values [1]. The ejected numbers of $\mathrm{Ru}$ sputtered particles was several hundred thousand.

The substrate positions were defined as a rotated angle the $\alpha$, in which the $\alpha$ exhibit relative angle from the position in front of each targets. The coordinate axes of X, $\mathrm{Y}$, and $\mathrm{Z}$ were selected in the direction of the moving substrate, the non-moving substrate, and the film thickness, respectively. Each time the transport path of sputtered particle was simulated corresponding with their

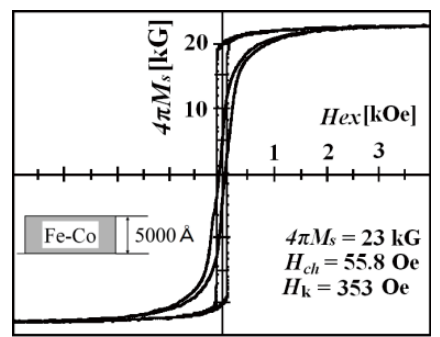

(a)

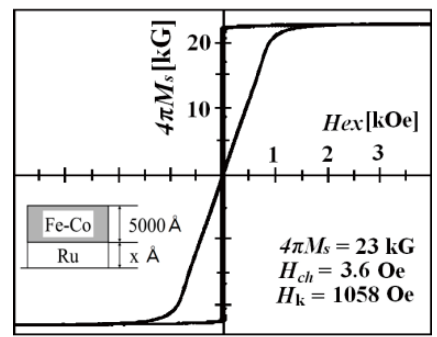

(b)

Fig.1 $M-H$ loops along easy and hard axies of magnetization for $\mathrm{CoFe}$ single layer (a), and $\mathrm{CoFe} / \mathrm{Ru}$ double layer (b), with the underlayer thickness of $20 \AA$. incident angle and energy to the substrate.

\section{EXPERIMENTAL RESLUT}

3.1 Effect of the underlayer on magnetostatic properties

Fig. 1 shows the $M-H$ loops of CoFe single layer (a), and $\mathrm{Co}-\mathrm{Fe} / \mathrm{Ru}$ double layer (b) with the underlayer thickness of $20 \AA . H_{k}$ is defined as the DC magnetic field. It was determined by the cross point where the easy magnetic axis direction of the magnetization curve and the tangent of hard magnetic axis direction of the magnetization curve in the film plane. A very higher $H_{k}$ of about $1000 \mathrm{Oe}$ was observed in the $\mathrm{Co}-\mathrm{Fe} / \mathrm{Ru}$ double layer. This value was about 3 times higher than that of the Co-Fe single layer. The underlayer was effective in the appearance of in-plane uniaxial magnetic anisotropy. This anisotropy field was 2-50 times higher than reported values of $\mathrm{Co}-\mathrm{Fe}$ based thin films [9-12]. The $M-H$ loops $\mathrm{Co}-\mathrm{Fe} / \mathrm{Ru}$ double layer show a well-defined uniaxial anisotropy, with a square, high-remanence in easy axis loop and a linearity, low coercivity in hard axis loop, as well as those in the reported papers [9-15]. It is expected that the film show a relative permeability of about 20 in the frequency was more than $10 \mathrm{GHz}$, according to the Landau-Lifshitz equation [15]. This change in the anisotropy field of $H_{k}$ was strictly depended on deposition of the underlayer.

3.2 Preferred orientation of (110) and surface structures of the underlayer

Preferred the crystalline orientation of the magnetic thin film is the important condition for the higher uniaxial- magnetic anisotropy field [15].

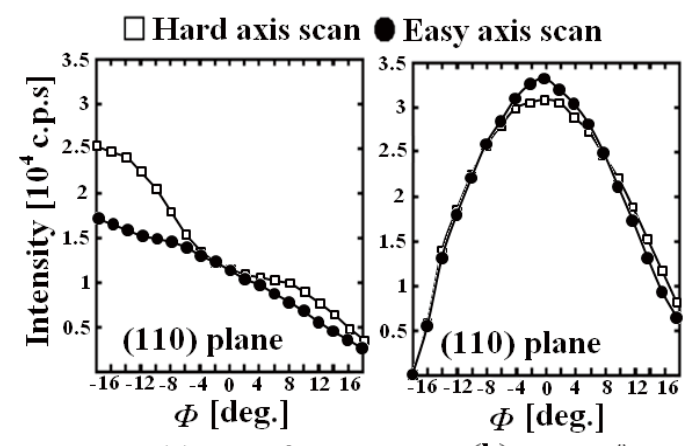

(a) Ru $0 \AA$

(b) Ru $20 \AA$

Fig. 2 (110) orientation profiles of $\varphi$ for $\mathrm{CoFe}$ single layer (a) and $\mathrm{CoFe} / \mathrm{Ru}$ double layer (b), with the underlayer thickness of $20 \AA$.

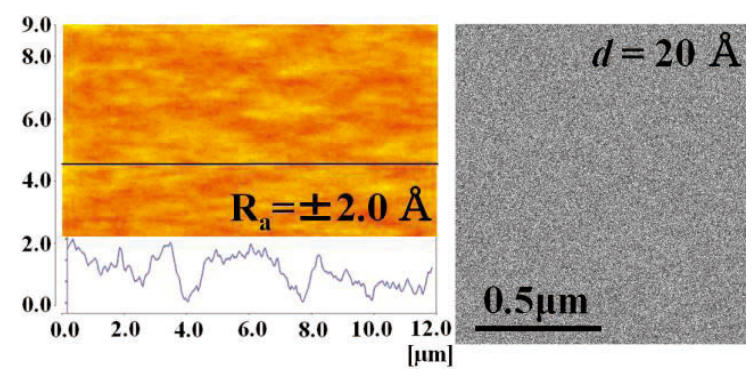

Fig.3 3DSEM surface image of the Ru underlayer and the scanning profile of asperity for $d=20 \AA$, before $\mathrm{CoFe}$ layer was deposited. 


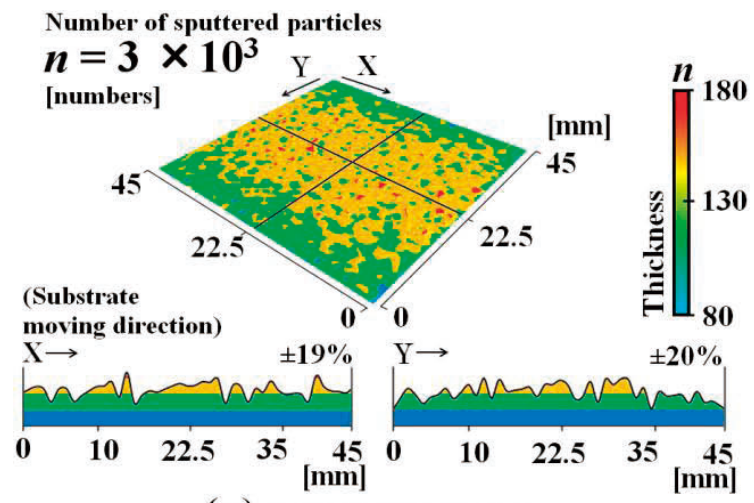

(a) Carrousel Sputtering

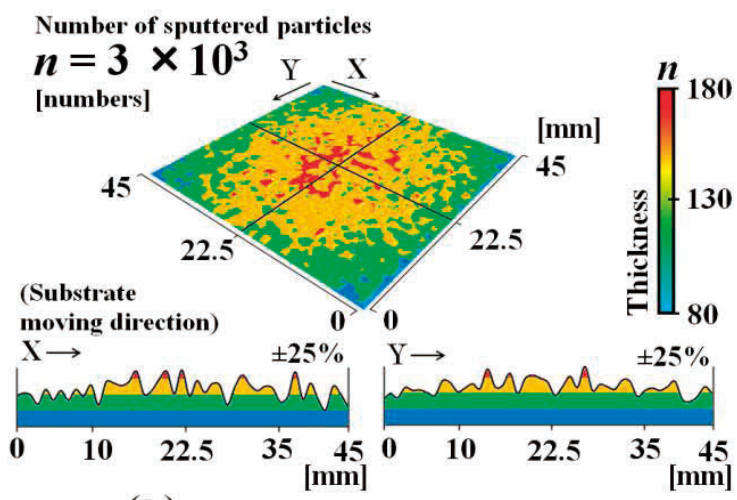

(b) Conventional RF Diode Sputtering

Fig. 4 Thickness distribution of Ru underlayer with its profiles in the direction of $\mathrm{X}$ and $\mathrm{Y}$ on the substrate, in the case of the carousel sputtering method (a) and the conventional RF diode sputtering (b).

Fig.2 shows (110) orientation profiles of $\phi$ for $\mathrm{CoFe}$ single layer (a) and $\mathrm{CoFe} / \mathrm{Ru}$ double layer (b), with the underlayer thickness of $20 \AA$, by using Schultz method.

In those profiles, $\phi$ means the angle (so-called $\alpha$ for pole figure) between normal directions to the film plane and those of each crystal plane, and the in-plane direction (so-called $\beta$ for pole figure) of the incident X-ray corresponds to the direction of the easy and the hard axis, respectively. In the Fig.2 (a) and (b), the underlayer caused a drastically change in (110) crystal plane orientation and a preferred orientation of (110) parallel with film plane, in which (110) orientation distributed within $\phi= \pm 16 \mathrm{deg}$. This (110) preferred orientation had been already explained that the reduction of surface energy due to the underlayer in the case of FeSi thin film materials [16].

The Fig. 3 shows 3DSEM surface image of the $\mathrm{Ru}$ underlayer and the scanning profile of asperity for $d=20$ $\AA$, before Co-Fe layer was deposited. Fine asperity was observed in the underlayers, the glass substrate was allover covered with the $\mathrm{Ru}$ layer, where isolated island structure was not observed. According to the observed scanning profile, surface roughness of the underlayers was $\pm 2.0-2.5 \AA$. The underlayer can be interpreted to have intrinsically low surface free energy.

\section{ANALYTICAL RESULTS AND DISCUSSION}

4.1 Thickness distribution and surface energy of $\mathrm{Ru}$ underlayer

Fig.4 (a) and (b) show thickness distribution of Ru underlayer with its profiles in the direction of $\mathrm{X}$ and $\mathrm{Y}$ on the substrate, in the case of the carousel sputtering method and the conventional RF diode sputtering, respectively. In the figure (a), the incident particles on the substrate were counted per $\alpha$. In the figure (b), the incident particles on the substrate were counted at the front of the target $\left(\alpha=0^{\circ}\right)$.

Generally the surface energy $\sigma_{0}$ is given by

$$
\sigma_{0}=\varphi / S \cdot \cdots \cdot \cdot \cdot \cdot \cdot(1)
$$

where $\varphi$ and $S$ are the bond energy and the area per an atom in a crystal plane in a solid, respectively. In this simulation, the surface energy $\sigma_{0}$ of the $\mathrm{Ru}$ underlayer was estimated by unevenness of the underlayer (surface area), because both (a) and (b) equal to the condition (atomic density, kind of the atoms, substrate temperature, and so forth) on the substrate.

The figure (a) shows the surface roughness of the underlayer agrees with experimental result as shown in figure 3 , which this roughness exhibited approximately flat as $\pm 19-20 \%$ in the direction of $\mathrm{X}$ and $\mathrm{Y}$. The values were less by $\pm 5-6 \%$ than those of the conventional RF diode sputtering (b).

It was indicated that the further reduction in surface energy of the Ru underlayer was induced by the carousel sputtering method, this surface energy was about $10 \%$ less than (b).

4.2 Incident energy of Ru particles and isotropic incident

Fig.5 shows energy of $\mathrm{Ru}$ incident particles and the substrate position $\alpha$. Energy values in case of $\alpha=0^{\circ}$ show distribution of incident energy for the conventional RF diode sputtering.

In the conventional RF diode sputtering $\left(\alpha=0^{\circ}\right)$, incident particles with $10 \mathrm{eV}$ energy, which was the same as the ejection energy due to no collision with $\mathrm{Ar}$ [6], existed $43 \%$ of the total number, because the mean free path of the sputtered particle was longer than distance between the target and the substrate. The other particles of $57 \%$ lost some energy by collision with gas (Ar)

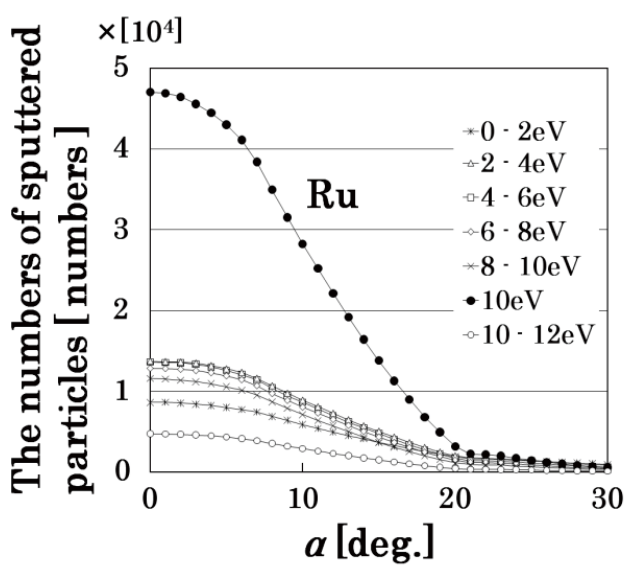

Fig.5 Energy of Ru incident particles and the substrate position $\alpha$. 


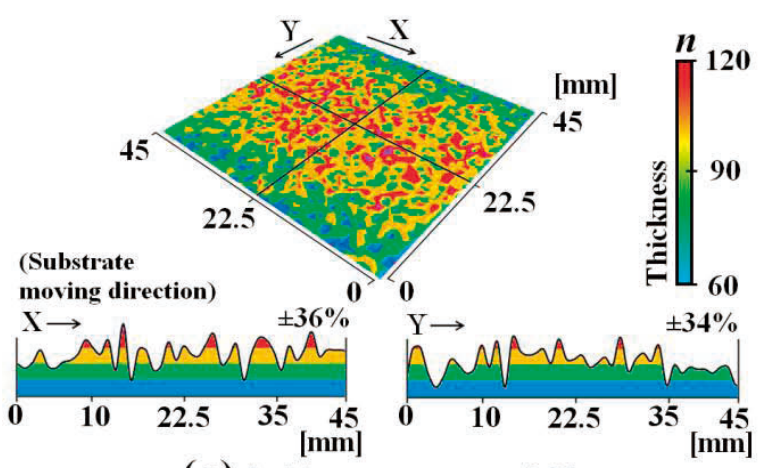

(a) Incident energy : $\mathrm{E}=\mathbf{1 0}[\mathrm{eV}]$

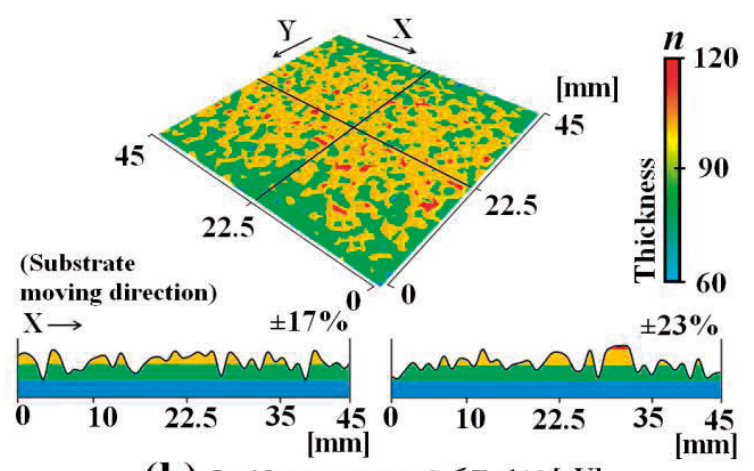

(b) Incident energy : $0 \leqq \mathrm{E}<10[\mathrm{eV}]$

Fig. 6 Thickness distribution of Ru underlayer with its profiles in the direction of $\mathrm{X}$ and $\mathrm{Y}$ on the substrate in the carousel sputtering method, (a) incident particles with high energy $(\mathrm{E}=10[\mathrm{eV}])$, and (b) incident particles with low energy $(0 \leqq \mathrm{E}<10[\mathrm{eV}])$.

particles, and they had isotropic incident in all areas on the substrate.

On the other hand, in the carousel sputtering method $\left(0^{\circ}<\alpha\right)$, number of low energy $(0 \leqq \mathrm{E}<10[\mathrm{eV}])$ particles increase up to $71 \%$ of the total number. It is because the collision probability between the Ru particle and Ar gas increases with increasing distance between the $\mathrm{Ru}$ target and the substrate.

It means that these low energy particles were remarkably related to the effect of the Ru underlayer.

\subsection{Low energy particles and surface energy}

Fig. 6 (a) and (b) show thickness distribution of $\mathrm{Ru}$ underlayer with its profiles in the direction of $\mathrm{X}$ and $\mathrm{Y}$ on the substrate in the carousel sputtering method, incident particles with high energy $(\mathrm{E}=10[\mathrm{eV}])$ and incident particles with low energy $(0 \leqq \mathrm{E}<10[\mathrm{eV}])$, respectively.

The surface roughness in case of (a) was larger than those of (b) by about 10-20\%. The surface energy in case of (b) was about $40 \%$ less than those of (a). It was found that the further reduction in surface energy of the $\mathrm{Ru}$ underlayer was induced by these low energy particles in the carousel sputtering method.

\section{CONCLUSION}

The nano-interface of the underlayer was effective in inducing the higher uniaxial anisotropy field for the $\mathrm{GHz}$ micro-device. We analyzed by using Monte Carlo simulation with respect to surface energy of the underlayer.

(1) In the carousel sputtering method, it was able to be simulated according with the experimental result that the $\mathrm{Ru}$ underlayer induce the low surface energy with the flatness in the film.

(2) It was found that this surface energy of $\mathrm{Ru}$ underlayer was less than that of the conventional RF diode sputtering.

(3) The low energy particles $(0 \leqq \mathrm{E}<10[\mathrm{eV}]: 71 \%$ of the total numbers) contributed to decrease the surface energy of Ru underlayer.

In this result, it was identified that the $\mathrm{Ru}$ nano-interface of the further reduction in surface energy brought the higher uniaxial-magnetic anisotropy for the $\mathrm{GHz}$ driving in MMIC.

\section{ACKNOWLEDGMENTS}

This work was supported in part by a Grant-in-Aid for Scientific Research (c) (2) (No.22560310) from the Japanese Ministry of Education, Culture, Sports, Science and Technology. The authors would like to thank Mr. K. Fukami and Mr. N. Yoshinaga for helpful discussion.

\section{REFERENCES}

[1] R.Imaizumi, M.Munakata, M.Ohkoshi, K.Maki, S.I.Aoqui, J.Magn.soc.Jpn., 34, 226 (2010).

[2] M.munakata, S.I.Aoqui, M.Ohkoshi, K.Maki, M.Sonehara, K.Ikeda, and T.Sato, J.Magn.soc.Jpn., 34, 248(2010).

[3] M. Ymaguchi, IEE J., 123, 716 (2003).

[4] K. Ikeda, K. Kobayashi, K. Ota, R. Kondo, T. Suzuki, and M. Fujimoto, IEEE, Trans. Magn., 39, 3057 (2003).

[5] M. Munakata, S. I. Aoqui, and M. Yagi, IEEE.Trans.Magn., 41, 3262 (2005).

[6] G.M.Turner, I. S. Falconer, B. W. James, and D. R. McKenzie, J. Appl. Phys., 65, 3671 (1989).

[7] T.Nakano, I.Mori, S.Baba, Appl.Surf.Sci., 113, 642 (1997).

[8] JMAG-Works, Commercial software: The Japan Research Institute, Ltd..

[9] N. X. Sun and S. N. Wang, J.Appl.Phys., 26, 1477 (2002).

[10] M. Munakata, M. Namikawa, M. Motoyama, M. Yagi, Y. Shimada, M. Yamaguchi, and K. I. Arai, Trans.Magn.Soc.Jpn., 2, 384(2002).

[11] B. D. Cullity, Elements of X-ray diffraction. Massachusetts, Addison - Wesley Pub. Co.(1977).

[12] S. H. Kong, T. Okamoto, and S. Nakagawa, IEEE.Trans.Magn., 39, 2285(2003).

[13] E. C. Stoner, and E. P. Wohlfarth, Phil.Trans.R.Soc.Lond., A240, 599(1948).

[14] M. Munakata, S. I. Aoqui, and M. Yagi, Mat.Sci.Eng., A, 413 (2005).

[15] G. Herzer, IEEE.Trans.Magn., 26, 1397(1997).

[16] Y. Shimada, K. Yamada, S. Hatta and H. Fukunaga, "Magnetic Matelials", Ed. By Y. Shimada, Koudan Sya, Tokyo (1999) pp.215

(Received January 31, 2011; Accepted April 19, 2011) 